\title{
The effect of periodic disturbances and carrying capacity on the significance of selection and drift in complex bacterial communities
}

\author{
Madeleine S. Gundersen $\mathbb{D}^{1 凶}{ }^{凶}$, lan Arthur Morelan ${ }^{2}$, Tom Andersen ${ }^{3}$, Ingrid Bakke ${ }^{1}$ and Olav Vadstein ${ }^{1}$ \\ (c) The Author(s) 2021, corrected publication 2022
}

Understanding how periodical disturbances affect the community assembly processes is vital for predicting temporal dynamics in microbial communities. However, the effect of dilutions as disturbances are poorly understood. We used a marine bacterial community to investigate the effect of disturbance (+/-) and carrying capacity (high/low) over 50 days in a dispersal-limited $2 \times 2$ factorial study in triplicates, with a crossover in the disturbance regime between microcosms halfway in the experiment. We modelled the rate of change in community composition between replicates and used this rate to quantify selection and ecological drift. The disturbed communities increased in Bray-Curtis similarity with $0.011 \pm 0.0045$ (Period 1) and $0.0092 \pm 0.0080$ day $^{-1}$ (Period 2), indicating that selection dominated community assembly. The undisturbed communities decreased in similarity at a rate of $-0.015 \pm 0.0038$ day $^{-1}$ in Period 1 and were stable in Period 2 at $0.00050 \pm 0.0040$ day $^{-1}$, suggesting drift structured community assembly. Interestingly, carrying capacity had minor effects on community dynamics. This study is the first to show that stochastic effects are suppressed by periodical disturbances resulting in exponential growth periods due to density-independent biomass loss and resource input. The increased contribution of selection as a response to disturbances implies that ecosystem prediction is achievable.

ISME Communications; https://doi.org/10.1038/s43705-021-00058-4

\section{INTRODUCTION}

Understanding how ecological assembly processes create temporal patterns in community composition is a major goal in community ecology [1]. After decades of debating whether community assembly follows neutral [2] or niche theory [3], it is now generally accepted that both stochastic and deterministic processes are important for community assembly $[1,4,5]$.

Four high-order processes structure community assembly. These are selection, ecological drift, dispersion, and diversification [4, 5]. These four processes have a varying degree of stochasticity and determinism. Selection is deterministic and based on differences in the fitness between populations. This process includes environmental filtering and biological interactions, such as competition and mutualisms. Drift is an entirely stochastic process that arises because there is a non-zero probability that an individual dies before it reproduces [6]. The outcome of drift is a change in the relative abundance of populations and can lead to local extinction if the abundance is low. Dispersion and diversification are two processes that are both stochastic and deterministic. Dispersion refers to an individual's movement from the regional to the local species pool, whereas diversification is the evolution of new strains [4]. The relative contribution of these four processes on community assembly can vary between sites and changes over time $[7,8]$.

Only experiments with high temporal resolution can evaluate the relative importance of these community assembly processes
$[9,10]$. During the last decade, studies using high temporal resolution sampling approaches have pointed to stochastic processes as being more important and selection as less important than previously assumed. This observation has been done in habitats such as bioreactors [11, 12], soil $[13,14]$, and wastewater treatment plants [15]. This increased awareness of stochasticity emphasises the need for more knowledge on temporal variation in the assembly processes.

A primary motivation for studying microbial community assembly is to understand the communities' responses to drivers affecting the high-order assembly processes in order to be able to forecast and manage them [10, 16]. Such control is vital in, for example, treating dysfunctional human gut microbiomes [17], ensuring stability during biological wastewater treatment [18] and providing an optimal microbial environment for fish in aquaculture [19].

Microbial communities often experience disturbances. Disturbances usually involve alterations in the available resources or the biomass concentration in the given environment. To predict the consequence of disturbances on the dynamics of microbial communities' it is essential to understand how the disturbance influences the four assembly processes' relative contributions $[7,10]$. Some studies have shown that disturbances affect the relative contribution of the assembly processes [11, 20-25], but conclusions vary depending on the disturbance type and the

\footnotetext{
${ }^{1}$ Department of Biotechnology and Food Science, NTNU - Norwegian University of Science and Technology, Trondheim, Norway. ${ }^{2}$ Independent researcher, Portland, OR, USA.
} ${ }^{3}$ Department of Biosciences, University of Oslo, Oslo, Norway. ${ }^{凶}$ email: madeleine.gundersen@ntnu.no 
ecosystem studied. Zhou et al. formulated two hypotheses describing the community assembly responses to disturbance based on the disturbance type [24]. Disturbances increasing the resource availability are suspected of enhancing the contribution of stochasticity [24] by weakening competition and strengthening priority effects (i.e., the effect of colonisation history) [7, 11, 24, 26]. Their hypothesis regarding biomass-reducing disturbances is contrasting, where deterministic processes should dominate mainly due to increased niche selection [24]. Thus, how disturbances that combine resource increase and biomass loss affect the community assembly is not known.

The maximum biomass an ecosystem can sustain is controlled by the carrying capacity. With regards to community assembly, carrying capacity can affect drift. Lower carrying capacities support lower biomasses, and as drift is density-dependent, more populations are vulnerable to extinction [6]. To our knowledge, no one has investigated how carrying capacity affects community assembly processes.

Microbial microcosms are excellent systems to study the effect of disturbances and carrying capacity on the temporal changes in community assembly. This is due to the short generation time of microorganisms, the potential for high experimental control and the possibility to include many experimental units [27]. In microcosms, one can eliminate dispersal, and if community composition is monitored by clustering 16s-rDNA sequences at a $97 \%$ similarity level, speciation is negligible [28]. Consequently, selection and drift are the only assembly processes shaping the bacterial communities [27].

Selection and drift can be quantified by investigating the similarity in community composition between biological replicates in systems without dispersal and speciation (Fig. 1). This approach assumes that if the selection is homogeneous (i.e., there is one stable equilibrium per condition), communities of replicate microcosms should over time become more similar if selection dominates and less similar if drift predominates. Moreover, if selection dominates, one expects the variation in community composition between replicate microcosms to decrease because the communities become more similar over time. Conversely, if drift dominates community assembly, replicates are expected to become less similar, and the variation in compositional similarity will increase with time.

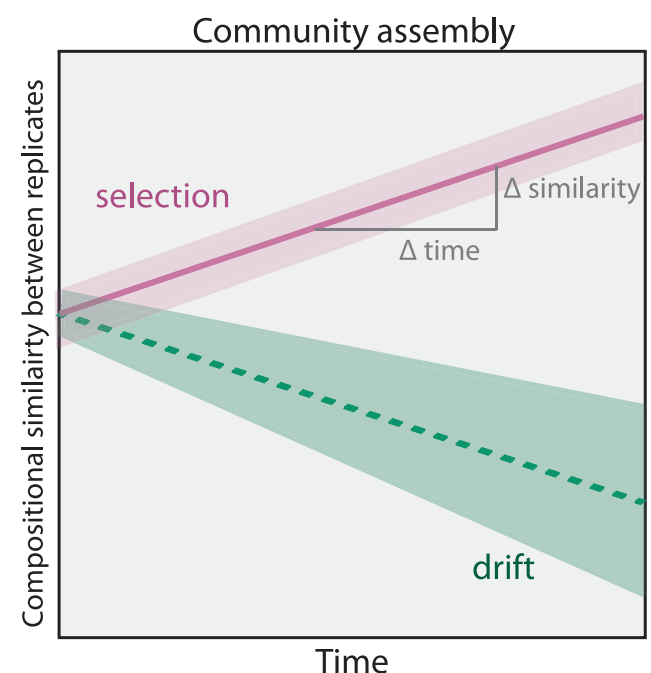

Fig. 1 A conceptual schematic of the temporal changes in community similarity between replicates if drift or selection dominates the community assembly. If selection dominates, the similarity between replicates increases over time, and the variance decrease or be stable. However, if drift dominates, replicates should become less similar over time, and the variance should increase.
In the present study, we aimed at disentangling the effects of disturbance introduced as periodic dilutions (undisturbed versus disturbed microcosms) and carrying capacity (high versus low) on succession and the relative importance of the assembly processes selection and drift. Specifically, we wanted to investigate the following research questions: (1) Do disturbances in the form of dilutions enhance selection or drift? (2) Does lower carrying capacities enhance the contribution of drift? (3) Are the effects of disturbances and carrying capacity dependent on the previous state of the communities?

To answer these questions, we used a $2 \times 2$ factorial crossover experimental design with three replicate microbial microcosms for each condition. The marine microbial communities were cultivated for 50 days either in chemostats or with semi-continuous cultivation with a 50-fold dilution every second day. The dilution functioned as a combined disturbance as it both reduced the community size and increased the specific resource supply. We quantified selection and drift using the approach described above, which allowed us to understand the effect of disturbance and carrying capacity on selection and drift.

\section{MATERIALS AND METHODS}

\section{Experimental design and sampling scheme}

A marine bacterial community was collected from sand-filtered water $(\sim 50$ $\mu \mathrm{m}$ ) collected from $70 \mathrm{~m}$ depth in the Trondheimsfjord, Norway (March 2018) and used to inoculate twelve microcosms $\left(500 \mathrm{~mL}\right.$, GLS $80^{\circ}$ stirred reactor, Duran, Germany) in a $2 \times 2$ factorial crossover design (Fig. 2). Each microcosm contained $250 \mathrm{~mL}$ culture that was stirred continuously (MIX 6, 2 mag AG, Germany), supplied with $0.2 \mu \mathrm{m}$ filtered (Millipore) hydrated air, and kept at $15^{\circ} \mathrm{C}$. The communities were cultivated in $f / 2$ medium [29] with either 0.33 (low carrying capacity, L) or $5 \times 0.33=1.67 \mathrm{mg} / \mathrm{L}$ (high carrying capacity, $\mathrm{H}$ ) of yeast extract, peptone and tryptone. The inorganic nutrients in the $f / 2$ media were 50 -fold diluted compared to the original recipe. The medium was either supplied continuously at a dilution rate of 1 day $^{-1}$ (Watson Marlow 520S peristaltic pump) or pulsed by a 1:50 dilution every second day equivalent to a continuous dilution rate of $\sim 2$ day $^{-1}$ (Fig. 2a, b). We define the pulsed communities as disturbed (D) and those continuously supplied with medium as undisturbed (U). On day $0,250 \mathrm{~mL}$ of the bacterial community was added to the undisturbed microcosms. In contrast, the communities in the disturbed microcosms were 1:50 diluted in sterile media upon inoculation to a final volume of $250 \mathrm{~mL}$. This disturbance regime was crossed after 28 days so that previously disturbed microcosms were undisturbed the last 22 days (DU) and vice versa (UD). The cultivation regimes are abbreviated as UDH, UDL, DUH, and DUL (Fig. 2c). Each cultivation regime was run in triplicates. The bacterial communities were sampled by filtering $\sim 30 \mathrm{~mL}$ of culture through a $0.2 \mu \mathrm{m}$ filter to a total of 206 samples ( 2 inoculum and 17 time-points $\times 4$ regimes $\times 3$ replicates) which were stored at $-20^{\circ} \mathrm{C}$ until further processing. Sampling of the disturbed communities was done right before the dilution.

\section{Extraction of bacterial DNA and 16S-rRNA amplicon sequencing}

Bacterial community DNA was extracted with the Qiagen DNeasy PowerSoil DNA extraction kit. The V3-V4 region of the bacterial 16S-rRNA gene was amplified using the broad-coverage primers with Illumina MiSeq adapter sequences III338F (5'-TCG-TCG-GCA-GCG-TCA-GAT-GTG-TAT-AAGAGA-CAG-NNN-NCC-TAC-GGG-WGG-CAG-CAG-3') and III805R (5'-GTC-TCGTGG-GCT-CGG-AGA-TGT-GTA-TAA-GAG-ACA-GNN-NNG-ACT-ACN-VGG-

GTA-TCT-AAK-CC- $\left.3^{\prime}\right)$. The reactions were run for 28 cycles $\left(98^{\circ} \mathrm{C} 15 \mathrm{~s}, 55^{\circ} \mathrm{C}\right.$ $20 \mathrm{~s}, 72^{\circ} \mathrm{C} 20 \mathrm{~s}$ ) with $0.3 \mu \mathrm{M}$ of each primer, $0.25 \mathrm{mM}$ of each dNTP, $1 \mathrm{mM}$ of $\mathrm{MgCl}_{2}, 2 \mu \mathrm{M}$ of $5 \times$ Phusion buffer HF, 0.015 units/ $\mu \mathrm{L}$ of Phusion Hot Start II DNA polymerase, $1 \mu \mathrm{L}$ of DNA template and $\mathrm{dH}_{2} \mathrm{O}$ to a total volume of 25 $\mu \mathrm{L}$. The amplicon library was prepared as described previously [30]. In brief, we used the SequalPrep Normalisation plate (96) kit (Invitrogen) to normalise and purify PCR products and the Illumina Nextera XT Index kits (FC-131-2001 and FC-131-2004) for amplicon indexing. The amplicon library was sequenced with V3 reagents by $300 \mathrm{bp}$ paired-end reads on two MiSeq Illumina runs at the Norwegian Sequencing Centre. Illumina sequencing data are deposited at the European Nucleotide Achieve (accession number ERS7182426-ERS7182513). 

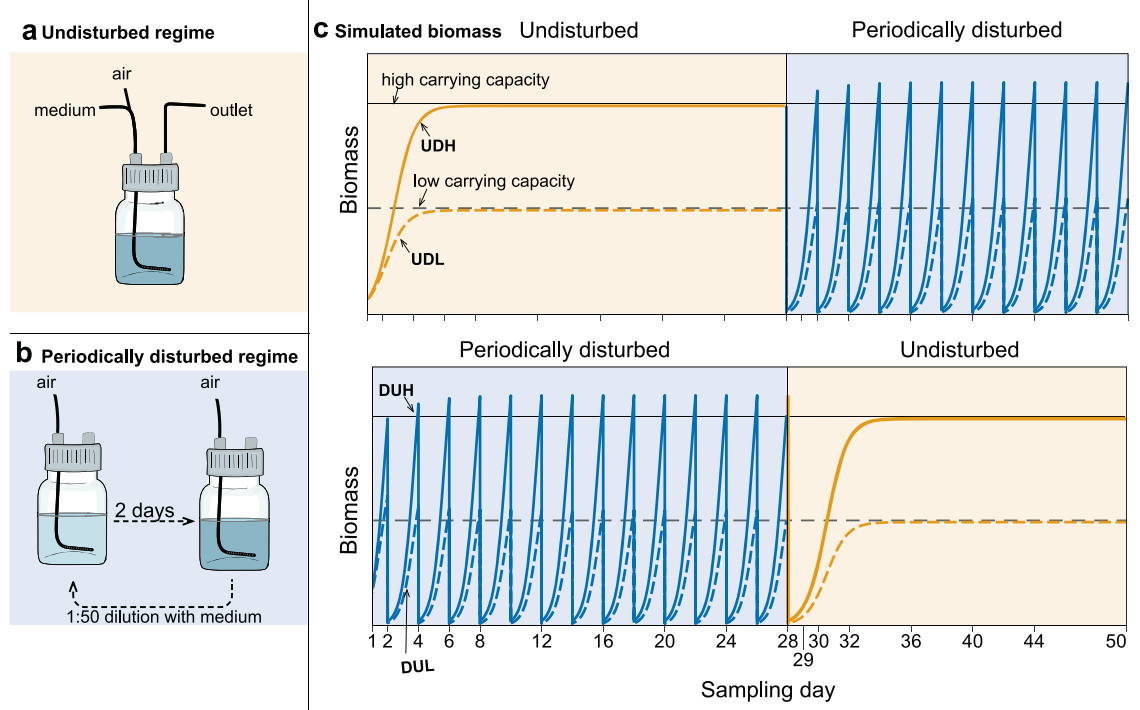

Fig. 2 Schematic overview of the experimental design. a Undisturbed communities $(U)$ received medium continuously at a dilution rate of 1 day $^{-1}$. b Disturbed communities (D) were 50-fold diluted with medium every second day. The dilution acted as a disturbance because the community's biomass was reduced substantially, and resources were introduced as a large pulse. c Simulated logistic growth of the microbial communities' biomass given the disturbance regime and carrying capacity (parameters: $\mu=2.5$ day $^{-1}$, continuous dilution $=1$ day ${ }^{-1}$ or semicontinuous 1:50 dilution every 2nd day). High carrying capacity is indicated as a solid black line, whereas low carrying capacity is presented as a dashed black line. When the communities are undisturbed, the biomass is expected to be at or near carrying capacity. The disturbance by dilution lowered the community's biomass by a factor of 50, bringing the community considerably below the carrying capacity, resulting in close to exponential growth between dilutions. Triplicate microcosms were operated over 50 days for each experimental condition, and the disturbance regime was switched after 28 days. The groups are abbreviated as UDH, UDL, DUH, and DUL, in which the first letter indicates the disturbance regime in period 1 (day 1-28), the second the disturbance regime in period 2 (day 29-50) and the third letter the carrying capacity of the media (high or low).

\section{Processing of sequence data}

We used the USEARCH pipeline (v11) to process the Illumina sequence data [31]. Briefly, using the command fastq_mergepairs, paired ends were merged, and primer sequences and reads shorter than $400 \mathrm{bp}$ were removed. The data was quality filtered using the command fastq_filter with an expected error parameter of 1 , and singletons were removed. We used the UPARSE-OTU algorithm to remove chimaeras and cluster OTUs at the $97 \%$ similarity level [32]. Taxonomy was assigned to the OTUs using the Sintax command with the RDP reference dataset (RPD training data set version 16$)$ at an $80 \%$ confidence threshold $[33,34]$.

\section{Analysis of diversity and differential abundance testing}

The resulting OTU-table was further analysed in R (version 3.6.1) [35]. Al R-code is provided at https://github.com/madeleine-gundersen/ disturcance-cc-assembly. We first evaluated the sequencing effort with the function rarecurve() in the vegan package (version 2.5-6) [36]. Then the data were normalised by averaging 1000 rarefied datasets created by randomly sub-sampling 10,000 reads without replacement using phyloseq_mult_raref() from the package metagMisc (version 0.0.4) (https:// github.com/vmikk/metagMisc/).

Alpha diversity was estimated as Hill diversity of order 0-2 [37] with the function renyi() in vegan. Bray-Curtis and Sørensen similarity indices were used to quantify beta diversity [38]. The variance in beta-diversity was ordinated with Principal Coordinate analysis (PCoA) [39]. Permutational multivariate analysis of variance (PERMANOVA) was used to test if sample groups significantly differed in community composition. The effect size of variables was evaluated with the $R^{2}$-value estimated with PERMANOVA [40]. To determine which OTUs increased in abundance due to the disturbance regimes, we performed a differential abundance test with DeSeq2 [41]. We used the non-normalised OTU-table as input to the DeSeq2 analysis. Only samples from the last 2 weeks of the cultivation periods were included in the analysis as PCOA ordinations indicated that the communities had stabilised. First, the abundance data were normalised using the median ratio method. DeSeq2 was then run with the Wald significance test assuming the negative binomial distribution. All $p$-values were FDR corrected.

\section{Estimation of selection and drift on community composition}

We developed a new approach to quantify the contribution of selection and drift during succession in highly controlled experimental settings where dispersal and speciation can be negligible (Fig. 1). Our approach is based on a three-step analytical process. First, the similarity in community composition between replicate pairs is calculated at each sampling day. Then the change in similarity is regressed again time. Finally, the slope of the temporal change in similarity is used to quantify selection and drift. Selection will result in communities becoming more similar with time, resulting in a positive or neutral slope. In contrast, drift causes communities to become less similar over time, manifested as negative slopes. In addition to the slope, the variation in similarity measurements can strengthen the conclusions as selection should decrease variation. In contrast, drift should increase the variation.

We calculated pair-wise community similarities between replicate microcosms at each sampling day, using Bray-Curtis and Sørensen similarity indices. In the following, we will use the term "replicate similarity" for this metric. We used a hierarchical Bayesian model approach to estimate the rate of change in the replicate similarity. We chose a Bayesian approach as it has the advantage of accounting for this dataset's hierarchical dependencies, few observations per time point and the observed heteroscedastic variance $[26,42]$.

We fitted hierarchical linear Bayesian models with replicate similarity as the dependent variable using the brms package (version 2.11.1) [43], which is a user-friendly front-end for the Stan system for Bayesian computing [44]. All models had a random intercept term for the three similarity comparisons ( $+(1 \mid$ comparison) in each time and regime combination. We modelled the replicate similarity by a normal distribution with fixed effects on both mean $(\mu)$ and standard deviation $(\sigma)$ using default priors. Fixed effects included 3-way interactions between time, disturbance, and carrying capacity for the mean model, whereas the standard deviation model only had interactions between time and disturbance. We meancentred the time variable to reduce correlations between fixed effect estimates. MCMC simulations with brms were run on 4 chains with 4000 samples each (2000 for warm-up), giving 8000 posterior samples. To reduce the number of divergent transitions in the MCMC sampling, we increased the value of the adapt_delta parameter to 0.99 (default is 0.95). 

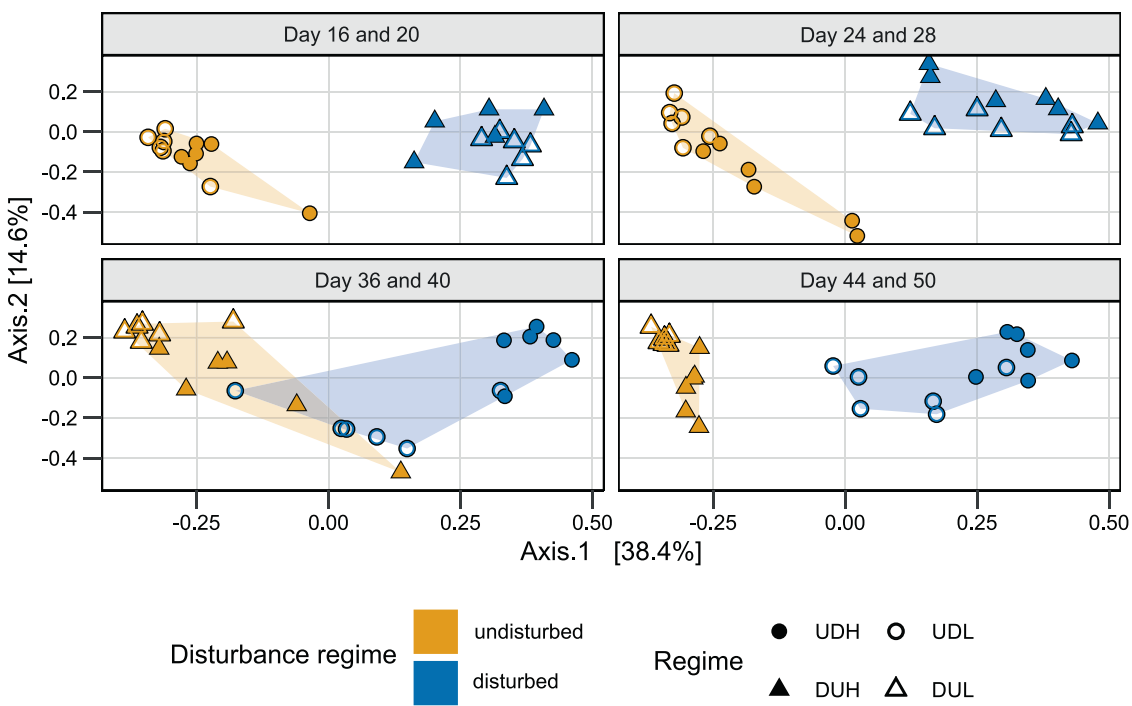

Regime

- UDH 0 UDL

$\Delta$ DUH $\triangle$ DUL

Fig. 3 PCoA ordination based on Bray-Curtis dissimilarity for the bacterial communities at the end of Period 1 (day 16-28) and Period 2 (36-50). The single ordination for these samples was split by sampling-week to highlight the succession based on the disturbance regime. UD (circles) were undisturbed the first 28 days and disturbed the last 22 days, while DU (triangles) were disturbed in the first period and undisturbed in the second. H (filled) and L (empty) indicates high and low carrying capacity, respectively. Colours represent the disturbance regime at sampling, and the shaded area the spread of samples with similar disturbance regimes.

We fitted several models, compared their predictive densities, and selected the model structure with the highest predictability for the temporal development of similarities between replicates. An overview of all estimated models and model selection process is given in the Supplementary material (Supplementary Figs. 1-2, Supplementary Tables 1-3).

We used the package tidybayes (version 2.0.3, http://mjskay.github.io/ tidybayes/) to extract posterior samples and the stat lineribbon() aesthetic from ggplot2 [45] to visualise fixed effect means and credible intervals of model predictions. As explained above, we interpreted community assembly as being dominated by selection if the time effect on the mean of replicate similarity was non-negative (i.e., $\mu$ day $^{-1} \geq 0$ ), and the standard deviation slope was non-positive $\left(\sigma\right.$ day $\left.^{-1}<0\right)$ (Fig. 1). Conversely, we interpreted a negative slope for the mean and a positive slope for the standard deviation as a community assembly dominated by drift (i.e., $\mu$ day $^{-1}<0, \sigma$ day $^{-1}>0$ ). In cases where the fit met neither of these criteria, we defined the community assembly as a mix of selection and drift.

\section{RESULTS}

To study the effect of the periodical disturbance and carrying capacity on community succession and the assembly processes, we cultured marine microbial communities under the DUH, DUL, $\mathrm{UDH}$, and UDL cultivation regimes and characterised their temporal dynamics using 16S-rDNA amplicon sequencing. The dataset contained a total of $12,945,783$ sequence reads with a mean of 63,460 reads ( $\pm 31,411$ SD) per sample. The dataset was normalised to 10000 reads per sample (Supplementary Fig. 3). The Hill alpha diversity of order 0,1 , and 2 of the normalised dataset correlated well with the non-normalised dataset $(p<0.05)$. The slopes of linear regressions between the alpha diversities of these datasets were close to one, indicating that the normalisedemulated the non-normalised dataset (Supplementary Fig. 4). The samples from the first sampling day were removed from the dataset because the richness dropped $43 \%$ from day 1 to 2 (Supplementary Fig. 5). This reduction was probably an adaption of the original seawater community to the culture conditions. During the rest of the experiment, the richness was relatively stable, and a total of 739 OTUs were observed for the normalised OTU table (Supplementary Fig. 5).

\section{The disturbance regime drove succession}

The community succession differed between the cultivation regimes, as indicated by $\mathrm{PCOA}$ ordinations based on both
Bray-Curtis (Fig. 3: day 16-28, 36-50, Supplementary Fig. 6: day 2-50) and Sørensen dissimilarities (Supplementary Fig. 7: day $16-28,36-50)$. Disturbance accounted for over 44 and $50 \%$ of the variation in Bray-Curtis dissimilarities at the end of Period 1 and 2, respectively ( $R^{2}$-effect size, $p<0.001$, PERMANOVA). Carrying capacity accounted for only $6(p=0.16)$ and $11 \%$ of the variation $(p=0.04)$ for the two periods. A fascinating observation was that switching the disturbance regime reversed the community succession from the undisturbed ordination space to the disturbed one and vice versa (Fig. 3, Supplementary Fig. 8). These ordinations indicated that disturbance was the main contributor to the succession and that carrying capacity had less effect.

Comparing the replicate similarity at the start and the end of each cultivation period indicated that the communities became more similar during disturbance than when undisturbed (Fig. 4a). For the disturbed communities, the Bray-Curtis similarity increased by $138 \%$ during Period 1 (DU) and $46 \%$ during Period 2 (UD). In contrast, the undisturbed communities decreased in similarity by $47 \%$ during Period 1 (UD) and increased by only $3.9 \%$ during Period 2 (DU, Fig. 4a). We investigated the replicate similarity change over time to determine whether selection or drift structured these successional patterns.

\section{Selection dominated during disturbance}

We used a Bayesian hierarchical model approach to estimate the replicate similarity change over time, and based on this, we examined whether selection or drift dominated the successions. The deterministic process selection should result in communities increasing in replicate similarity over time. This increased similarity will also result in a decrease in variation between similarity measurements. In contrast, the random process drift would reduce the replicate similarity and increase the variation over time (see Fig. 1 and Materials and method for more information).

For the disturbed communities in Period 1 (DU), the posteriordistributions of the model parameters revealed that the replicate similarity increased with $0.011 \pm 0.0045$ day $^{-1}$ (mean \pm SD), whereas the standard deviation decreased $0.054 \pm 0.010$ day $^{-1}$ (Fig. 4b, c). This increased replicate similarity and decreased standard deviation over time indicate that selection was the dominating assembly process (Fig. 5a). Moreover, we observed the same trends for the disturbed communities in Period 2 (UD) with 

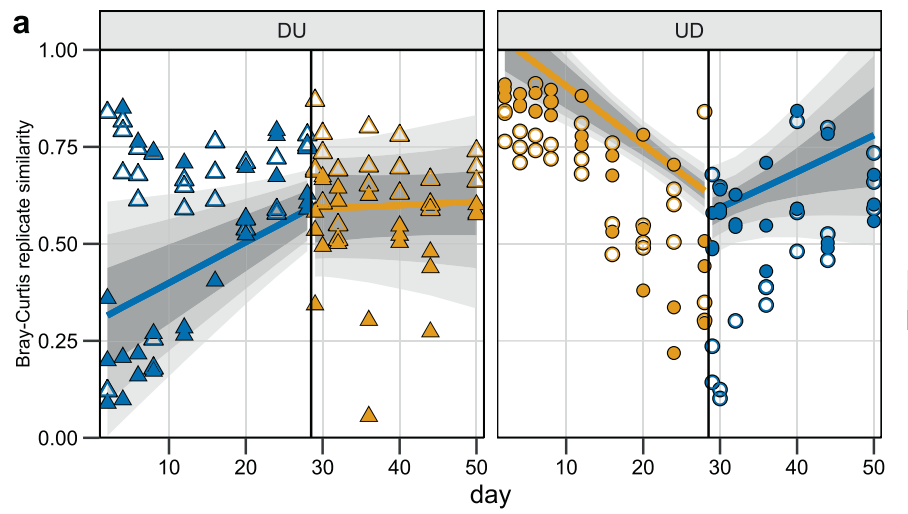

Credible interval

0.95

0.8
0.5

Disturbance regime

- undisturbed

- disturbed

b

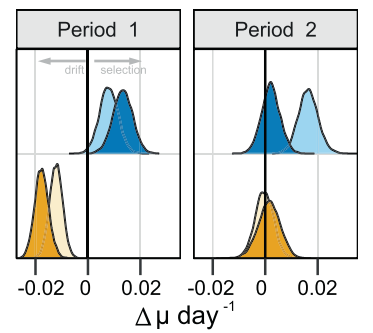

c
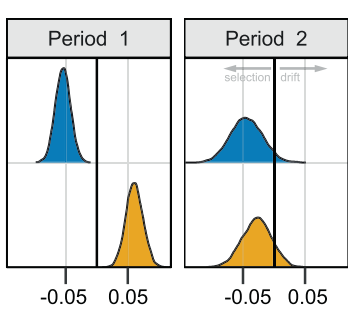

Regime

$:$ UDH
O UDL
A DUH
$\Delta$ DUL

$\Delta \log (\sigma)$ day $^{-1}$

Fig. 4 The Bray-Curtis based models and coefficient estimates for the change in the similarity between replicates over time. a The similarity between replicate communities as a function of time. The models for the replicate similarity change over time are presented as lines with the $0.5,0.8$, and 0.95 credible intervals around it. The observed data used as the response variable in the models are presented as points. UD (circles) were undisturbed the first 28 days and disturbed the last 22 days, while DU (triangles) were disturbed in the first period and undisturbed in the second. $\mathrm{H}$ (filled) and L (empty) indicates high and low carrying capacity, respectively. Colours represent the disturbance regime at sampling. $\mathbf{b}$ The posterior distributions of the expected replicate similarity $(\mu)$ change per day given the interaction between time, the disturbance regime and carrying capacity. The distribution reflects all 8000 estimated replicate similarity changes that would give the observed data. Light and dark colours indicate low and high carrying capacity, respectively. The colour indicates the disturbance regime at sampling. c The posterior distributions for the change in standard deviation per day given the interaction between time and disturbance regime. The distribution reflects all 8000 estimated standard deviation changes per day that would give the observed data. The colour indicates the disturbance regime at sampling.
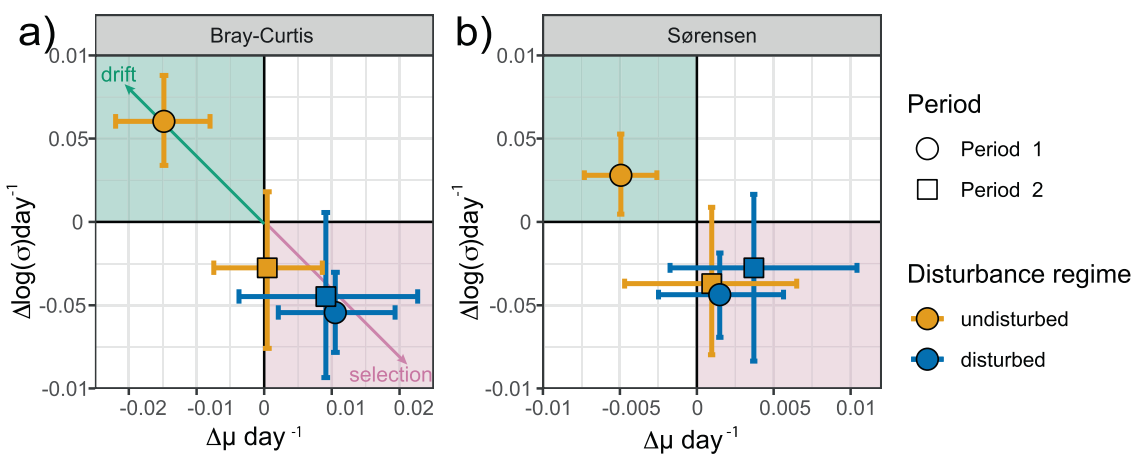

Fig. 5 Model estimates for the replicate similarity and change in standard deviation. The mean change in replicate similarity $(\mu)$ over time and mean change in standard deviation $(\sigma)$ (points) and the accompanying $95 \%$ credible intervals for each estimate, as inferred using the Bayesian hierarchical model approach, based on a Bray-Curtis and $\mathbf{b}$ Sørensen based models. The green area indicates the coordinate space where drift dominates, while the pink areas indicate where selection is dominating. Point colour indicates the disturbance regime and shapes the cultivation period.

an increase in replicate similarity of $0.0092 \pm 0.0080$ day $^{-1}$ and a decrease in the standard deviation of $-0.044 \pm 0.025$ day $^{-1}$ (Fig. 4b, c). This coherent observation strengthens the conclusion that selection dominated community assembly during disturbances.

The modelling results were different for the undisturbed communities. During Period 1 (UD), the replicate similarity rate decreased by $-0.015 \pm 0.0038$ day $^{-1}$ and had a temporal increase in variation of $0.061 \pm 0.014$ day $^{-1}$ (Fig. $4 \mathrm{~b}, \mathrm{c}$ ), indicating that drift dominated the community assembly (Fig. 5a). For the communities that switched from a disturbed to an undisturbed regime
(DU) in Period 2 the dominating assembly process was less obvious. The replicate similarity rate was relatively stable with a mean of $0.00050 \pm 0.00400$ day $^{-1}$ and a decrease in the standard deviation of $-0.028 \pm 0.024$ (Fig. 4b, c). These values categorise the assembly as selection (Fig. 5). However, comparing the replicate similarity rate of the communities from Period 1 to the one in Period 2 shows that the rate decreased substantially. The average similarity rate transitioned from the selectioncoordinate space towards the one where drift dominates (Fig. 5a).

The results were similar for models based on the Sørensen similarity, with an overall increase in replicate similarity over time 


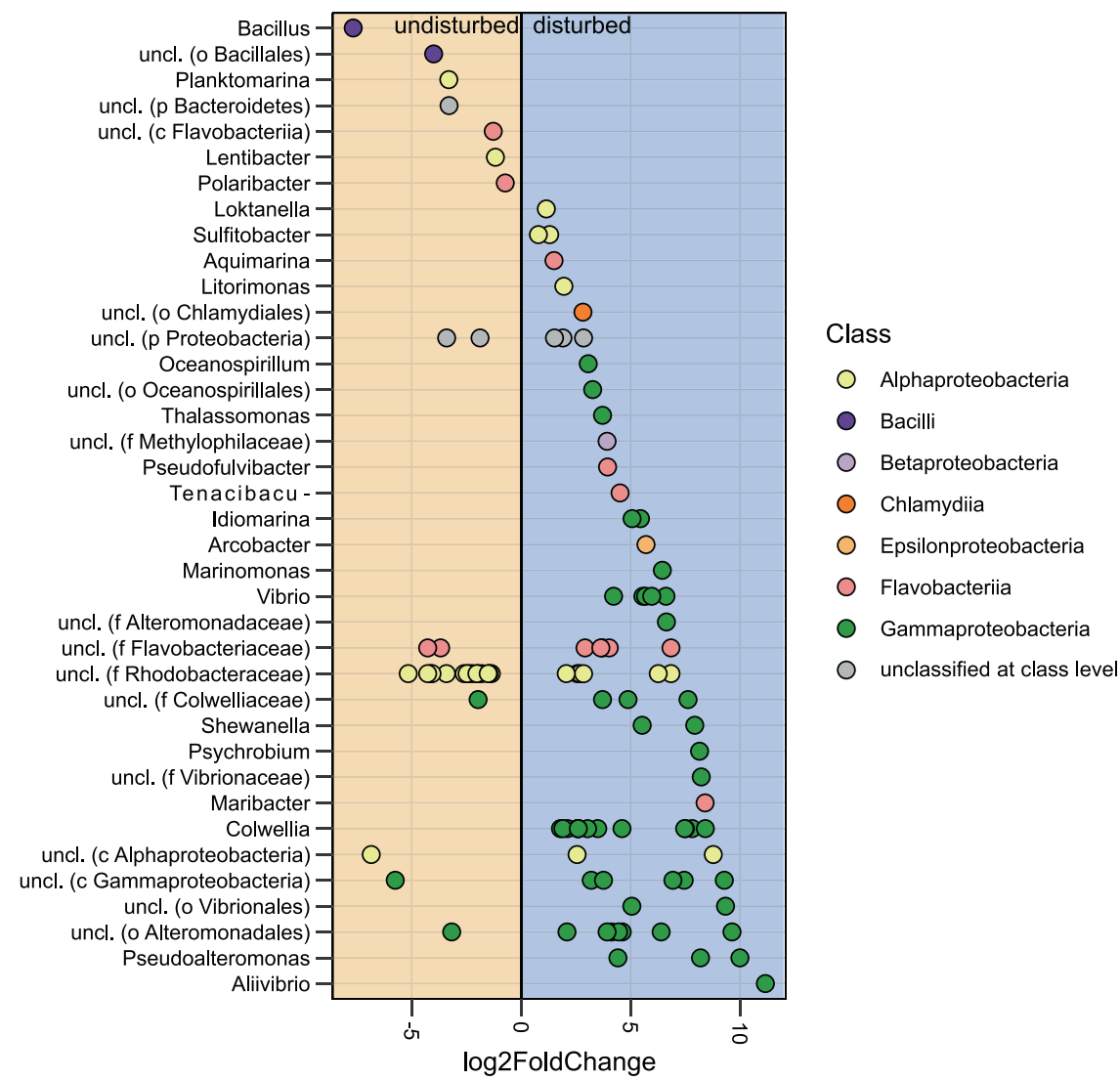

Fig. 6 The log 2 fold change in relative abundance between the disturbed and undisturbed communities during the last 2 weeks of the cultivation periods (week 3-4 and 6-7). Only OTUs with a significance level lower than 0.05 are shown (FDR-adjusted DESeq2 $p$-values). Each point represents an OTU coloured by the class classification. OTUs were grouped according to the genus level. The lowest taxonomic classification obtained is indicated in parenthesis for OTUs that could not be classified at the genus level (p phylum, c class, o order, $\mathrm{f}$ family, $\mathrm{g}$ genus). OTUs with higher abundance during disturbance are in the blue shaded area, whereas those with higher abundance when the environment was undisturbed are in the orange shaded area.

for the disturbed regimes of $0.0015 \pm 0.0021$ day $^{-1}$ in Period 1 (DU) and $0.0041 \pm 0.0040$ day $^{-1}$ in Period 2 (UD), and a decrease in the standard deviation of the replicate similarity over time (Fig. 5b, Supplementary Fig. 9). For the undisturbed communities, there was a slight temporal decrease in Sørensen replicate similarity at a rate of $-0.0049 \pm 0.0012$ day $^{-1}$ in Period 1 (UD), whereas in Period 2 there was an insignificant change in replicate similarity $(0.00096$ \pm 0.0029 day $^{-1}$; DU). These results supported the findings based on the Bray-Curtis similarity; drift dominated assembly for the undisturbed communities, whereas selection dominated when the communities were disturbed.

\section{Gammaproteobacteria increased in relative abundance during disturbance}

The PCOA ordination and the replicate similarity models showed that the disturbance regime impacted the assembly processes. We performed a DeSeq2 differential analysis to elucidate which OTUs had significantly different abundances between the disturbed and undisturbed regime. This analysis revealed that 107 of the 535 OTUs contributed significantly $(p<0.05)$ to differences in community composition between the disturbed and the undisturbed regimes. These OTUs were grouped at the genus level (Fig. 6). Interestingly, around $60 \%$ of these genera included only one OTU. For genera with more OTUs affected, the general trend was that the OTUs responded similarly to the disturbance regime (i.e. either positive or negative fold change in relative abundance). For example, all 13 OTUs classified as Colwellia and all 5 OTUs classified as Vibrio had higher abundance during disturbance. However, this was not the case for all the groups. For example, of the 21 OTUs classified to Rhodobacteraceae, 6 were in higher abundances during the disturbed periods, whereas 15 were more abundant during undisturbed periods. Thus, some genera's OTU abundances appeared to respond to the disturbance regime coherently, whereas others did not. Of the 107 OTUs significantly affected by the disturbance regime, $72 \%$ had increased abundances when the environment was disturbed. Especially noteworthy was the Gammaproteobacteria, where $94 \%$ of the OTUs significantly affected by the disturbance regime had higher abundances during disturbance with up to an 11.2 fold-change.

\section{DISCUSSION}

Predicting community responses to ecosystem changes is essential for improving ecosystem management. From an industrial perspective, we are dependent on stable microbial communities that perform well. Moreover, we live in a time where humans create disturbances at various levels in natural ecosystems. It is therefore important to comprehend the consequences of our activity. To predict the community response to external forces, we need to understand how different ecosystems affect the community assembly processes.

We aimed to fill the knowledge gap on how carrying capacity and periodical disturbances affect the community assembly. It has previously been shown that the carrying capacity affects the community composition [46]. However, its effect on the assembly processes has remained unclear. Ecosystems with a lower carrying capacity support lower community size. Because the outcome of 
drift is density-dependent [6], communities with a low carrying capacity should have more populations vulnerable to drifting to extinction. However, our five-times difference in carrying capacity between cultivation regimes did not result in apparent differences in community assembly. The only exception was for the disturbed communities in Period 2, where the low carrying capacity regime (UDL) indicated a stronger influence of selection than the high (UDH; Fig. 4b). This observation was surprising as we hypothesised that drift might be more pronounced in systems with lower carrying capacity. In conclusion, the minor effects of carrying capacity observed for the replicate similarity rate for the undisturbed communities suggest that the effect of carrying capacity should be investigated further, including larger differences in carrying capacity.

The effect of the disturbance regime on the microbial community assembly was more evident. The disturbance we investigated was a substantial dilution of the microcosm's inoculum. The dilution has two significant effects: the community size is reduced, and the concentration of resources increases strongly for the remaining individuals. These two changes are relevant in natural and human-created ecosystems, where resource supply vary due to natural processes (e.g. patchiness and floods) and human activity (e.g. eutrophication and saprobiation).

Investigating the temporal community composition through ordinations can reveal overall successional trajectories [47]. We found that whereas the PCoA ordinations indicated an overall deterministic trajectory for the undisturbed communities, the replicate similarity rate indicated that drift dominated the community assembly. This was evident for the microcosms starting with undisturbed culture conditions (UD $\Delta \mu>0$; Fig. 5). However, the results were less evident for the communities going from disturbed to undisturbed conditions (DU) as the replicate similarity rate was around zero. Nonetheless, there was an apparent decrease in the replicate similarity rate when going from disturbed $\left(\Delta \mu 1.1 \times 10^{-2}\right)$ to undisturbed conditions $\left(\Delta \mu 5 \times 10^{-4}\right)$.

The strength and unique feature of our experiment is the crossed design of the disturbance regimes. This crossing considerably increases the robustness of the conclusions drawn from the data. First, during the first period, all microcosms were inoculated with the same community, but in the second period, the twelve communities had assembled individually for 28 days. We could therefore investigate the effects of our experimental variables on drift and selection with different starting conditions. The temporal trends in the data were found to be independent of the starting condition, substantially increasing the strength of our conclusion.

Second, subjecting the communities to the opposite disturbance regime in Period 2 supports that we had stable attractors in our systems. An attractor is a point or a trajectory in the state space of a dynamical system. If the attractor is locally stable, the system will tend to evolve toward it from a wide range of starting conditions and stay close to it even if slightly disturbed [48]. We observed locally stable attractors based on the disturbance regime and thus one stationary phase for each disturbance regime. Some ecological systems show dramatic regime shifts between alternative stationary states in response to changes in an external driver [49]. Such systems typically exhibit hysteresis in the sense that they will not return directly to the original state by an opposite change in the driver. We found that community composition was reversible and dependent on the disturbance regime, as highlighted by the Bray-Curtis ordinations (Fig. 4). This reversibility indicates that the community changes we observed were not catastrophic bifurcations or regime shifts and that it is unlikely that the systems contain multiple stationary states within the same disturbance regime. We think this gives strong support for assuming that drift is the main driver for divergence in the community composition and that selection towards alternative attractors probably plays a minor role. Thus, we can conclude that shifting from a disturbed to an undisturbed ecosystem increased the contribution of drift. Our observations corroborate other investigations of bioreactors $[15,50]$ and simulations [51] that report that stochasticity is fundamental for the assembly of communities. However, the finding that drift was important for structuring the undisturbed microcosms was unexpected.

In dispersal-limited communities where resources are supplied continuously, such as in the undisturbed communities examined here, the selective process competition has been hypothesised to be high [7]. However, our experimental environment offered little variation in the resources provided, as the medium provided was the same throughout the experiment. This may have led to populations becoming "ecologically equivalent", meaning that their fitness difference was too small to result in competitive exclusion on the time scale of our experiment $[5,52]$. Under these assumptions, community assembly is similar to the neutral model in which the growth rates of the community members are comparable [53].

During disturbances, we found that selection dominated community assembly. Our results support Zhou et al. hypothesis stating that determinism should increase due to biomass loss in dispersal-limited communities [24]. However, they oppose their other hypothesis stating that nutrient inputs should increase stochasticity [24], making low abundant populations vulnerable to local extinction [6,7]. During the disturbances, the Sørensen similarity between replicates was stable or increasing, indicating that the periodical disturbance did not result in the extinction of low abundant populations. Instead, it appears that the dilution removed competition for some time, resulting in a phase where all populations got "a piece of the cake". Several studies have observed increased stochasticity as a result of increased resource availability $[7,11,24,26]$. However, we found that disturbances resulting in periods with exponential growth due to densityindependent loss of individuals and high resource input suppressed the effect of stochastic processes. This exponential growth period without competition would enable more populations to stay above the detection limits of the 16S-rDNAsequencing method.

More OTUs were enriched under the disturbed regime than under the undisturbed. During the disturbance, the microcosms were diluted $\sim 2$ day $^{-1}$, whereas the dilution factor was 1 day $^{-1}$ during the undisturbed regime. We cannot assume steady-state in the disturbed microcosms, but it was interesting to see a substantial increase in the abundance of OTUs classified as Gammaproteobacteria. Gammaproteobacteria include many opportunists [54] that appeared to exploit the resource surplus following the disturbance. This opportunistic lifestyle fits within the $\mathrm{r}$ - and K-strategist framework [55].

Organisms with high maximum growth rates but low competitive abilities are classified as $r$-strategists. These $r$-strategists are superior in environments where the biomass is below the carrying capacity. On the other hand, K-strategists are successful in competitive environments due to their high substrate affinity and resource specialisation [56]. Based on the taxonomic responses, it appears as disturbances in the form of dilutions selected for $r$-strategists, whereas the undisturbed regime selected for K-strategists. The r-strategists selected for during the disturbance periods included genera such as Vibrio and Colwellia [57], and the genus Vibrio includes many pathogenic strains [58]. Thus, our findings may have implications for land-based aquaculture systems where conditions favouring $r$-strategists is linked to high mortality and reduced viability of fish [56].

The DeSeq 2 results pose some new questions regarding the link between phylogeny and niche fitness. Generally, ecologists assume that closely related taxa have similar niches, as they have a common evolutionary history and, thus, similar physiology 
$[59,60]$. For example, here, OTUs belonging to Gammaproteobacteria co-occurred when the environment was disturbed. However, for other classes such as Alphaproteobacteria and Flavobacteria, the OTUs responded differently to the disturbance regimes, despite belonging to the same class. This lack of phylogenetically coherent response indicates that the paradigm of correlation between phylogeny and niche requires further studies.

This study was performed on complex marine microbial communities cultivated under controlled experimental conditions. We found that undisturbed environments enhanced the contribution of drift on community assembly and that disturbances increased the effect of selection. These observations might be different in more diverse ecosystems such as soils or the human gut. In such ecosystems, the microbes are more closely associated with, for example, soil particles or attached to the gut lining. It has been shown that the biofilm-associated and planktonic microbial communities have different community compositions [61]. Consequently, the community assembly processes may be affected differently by environmental fluctuations. Our experimental variables should therefore be tested in other ecosystem settings to verify our conclusions.

To our knowledge, this study is the first to experimentally estimate the effect of periodical disturbances and carrying capacity on community assembly in dispersal-limited ecosystems. We observed that carrying capacity had little effect on community assembly and that undisturbed communities were structured more by drift than disturbed systems dominated by selection. Using an experimental crossover design for the disturbance regime, we showed that these observations were independent of the initial community composition. Our experiment illustrates that cultivating complex natural microbial communities under lab conditions allowed us to test ecologically relevant system variables and draw robust conclusions.

\section{REFERENCES}

1. Zhou J, Ning D. Stochastic community assembly: does it matter in microbial ecology? Microb Mol Biol Rev. 2017;81:e00002-17.

2. Hubbell SP. The unified neutral theory of biodiversity and biogeography. Princeton New Jersey: Princeton University Press; 2001.

3. Chesson P. Mechanisms of maintenance of species diversity. Ann Rev Ecol Syst. 2000;31:343-66.

4. Vellend M. Conceptual synthesis in community ecology. Quarterly Rev Biol. 2010;85:183-206.

5. Nemergut DR, et al. Patterns and processes of microbial community assembly. Microbiol Mol Biol Rev. 2013;77:342-56.

6. Vellend M. The theory of ecological communities. New Jersey: Princeton University Press, Princeton; 2016. p. 57.

7. Dini-Andreote F, Stegen JC, van Elsas JD, Salles JF. Disentangling mechanisms that mediate the balance between stochastic and deterministic processes in microbial succession. Proc Natl Acad Sci. 2015;112:E1326 LP-E1332.

8. Langenheder S, Berga M, Östman Ö, Székely AJ. Temporal variation of $\beta$-diversity and assembly mechanisms in a bacterial metacommunity. ISME J. 2012;6:1107-14.

9. Gonzalez A, et al. Characterizing microbial communities through space and time. Curr Opin Biotechnol. 2012;23:431-6.

10. De Vrieze J, et al. Stochasticity in microbiology: managing unpredictability to reach the Sustainable Development Goals. Microbial Biotechnol. 2020;13:829-43.

11. Zhou J, et al. Stochastic Assembly Leads to Alternative Communities with Distinct Functions in a Bioreactor Microbial Community. mBio. 2013;4:e00584-12.

12. Liébana $R$, et al. Combined Deterministic and Stochastic Processes Control Microbial Succession in Replicate Granular Biofilm Reactors. Environ Sci Technol. 2019;53:4912-21.

13. Rigonato J, et al. Temporal assessment of microbial communities in soils of two contrasting mangroves. Brazilian J Microbiol. 2018;49:87-96.

14. Guo $X$, et al. Climate warming leads to divergent succession of grassland microbial communities. Nat Climate Change. 2018;8:813-8. 2018 8:9

15. Ofițeru ID, et al. Combined niche and neutral effects in a microbial wastewater treatment community. PNAS. 2010;107:15345-50.
16. Rittmann BE. Microbial ecology to manage processes in environmental biotechnology. Trends Biotechnol. 2006;24:261-6.

17. Shafquat $A$, Joice $R$, Simmons $S L$, Huttenhower $C$. Functional and phylogenetic assembly of microbial communities in the human microbiome. Trends Microbiol. 2014;22:261-6.

18. Graham DW, Smith VH. Designed ecosystem services: application of ecological principles in wastewater treatment engineering. Designed Ecosyst Serv. 2004;2:199-206.

19. Vadstein O, et al. Managing the microbial community of marine fish larvae: a holistic perspective for larviculture. Front Microbiol. 2018;9:1820.

20. Chase JM. Drought mediates the importance of stochastic community assembly. PNAS. 2007;104:17430 LP-17434.

21. Herold $M$, et al. Integration of time-series meta-omics data reveals how microbial ecosystems respond to disturbance. Nat Commun. 2020;11:1-14.

22. Vass $M$, Székely AJ, Lindström ES, Langenheder $S$. Using null models to compare bacterial and microeukaryotic metacommunity assembly under shifting environmental conditions. Scientific Reports. 2020;10:1-13.

23. Fillinger L, Hug K, Griebler C. Aquifer recharge viewed through the lens of microbial community ecology: Initial disturbance response, and impacts of species sorting versus mass effects on microbial community assembly in groundwater during riverbank filtration. Water Res. 2021;189:116631.

24. Zhou J, et al. Stochasticity, succession, and environmental perturbations in a fluidic ecosystem. PNAS. 2014;111:E836-45.

25. Ferrenberg $\mathrm{S}$, et al. Changes in assembly processes in soil bacterial communities following a wildfire disturbance. ISME J. 2013;7:1102-11.

26. Shade $A$ et al. Fundamentals of microbial community resistance and resilience. Front Microbiol. 2012;3:417.

27. Jessup $C M$, et al. Big questions, small worlds: microbial model systems in ecology. Trends Ecol Evol. 2004;19:189-97.

28. Burns AR, et al. Contribution of neutral processes to the assembly of gut microbial communities in the zebrafish over host development. ISME J. 2015;10:655-64.

29. Smith WL, Chanley MH Culture of marine invertebrate animals: proceedings of the conference on culture of marine invertebrate animals. New York: Plenum Press; 1975.

30. Vestrum RI, Attramadal KJK, Vadstein O, Gundersen MS, Bakke I. Bacterial community assembly in Atlantic cod larvae (Gadus morhua): contributions of ecological processes and metacommunity structure. FEMS Microbiol Ecol. 2020;96:1-12.

31. Edgar RC. Search and clustering orders of magnitude faster than BLAST. Bioinformatics. 2010;26:2460-1.

32. Edgar RC. UPARSE: highly accurate OTU sequences from microbial amplicon reads. Nat Methods. 2013;10:996-8.

33. Cole JR, et al. Ribosomal Database Project: data and tools for high throughput rRNA analysis. Nucleic Acids Res. 2013;42:D633-42.

34. Edgar RC. SINTAX: a simple non-Bayesian taxonomy classifier for $16 \mathrm{~S}$ and ITS sequences. bioRxiv. 2016;74161.

35. $\mathrm{R}$ Core Team. R: A language and environment for statistical computing. $\mathrm{R}$ Foundation for Statistical Computing, Vienna, Austria. 2020. https://www.Rproject.org/.

36. Dixon P. VEGAN, a package of R functions for community ecology. J Vegetation Sci. 2003;14:927-30.

37. Lucas $\mathrm{R}$ et al. A critical evaluation of ecological indices for the comparative analysis of microbial communities based on molecular datasets. FEMS Microbiol Ecol. 2017;93:1-15.

38. Legendre $P$, Cáceres Mde. Beta diversity as the variance of community data: dissimilarity coefficients and partitioning. Ecol Lett. 2013;16:951-63.

39. Ramette A. Multivariate analyses in microbial ecology. FEMS Microbiol Ecol. 2007;62:142.

40. Anderson MJ. A new method for non-parametric multivariate analysis of variance. Austral Ecol. 2001;26:32-46.

41. Love MI, Huber W, Anders S. Moderated estimation of fold change and dispersion for RNA-seq data with DESeq2. Genome Biol. 2014;15:1-21.

42. Carpenter SR, Brock WA. Rising variance: a leading indicator of ecological transition. Ecol Lett. 2006;9:311-8.

43. Bürkner P-C. brms: An R Package for Bayesian Multilevel Models Using Stan. J Stat Softw. 2017;80:1-28.

44. Stan Development Team. YEAR. Stan Modeling Language Users Guide and Reference Manual, VERSION. https://mc-stan.org.

45. Wickham H. ggplot2: Elegant Graphics for Data Analysis. New York: SpringerVerlag; 2016. vol 2. p. 1-266.

46. Attramadal KJK, et al. Microbial maturation of intake water at different carrying capacities affects microbial control in rearing tanks for marine fish larvae. Aquaculture. 2016;457:68-72. 
47. Fukami T, Bezemer TM, Mortimer SR, Putten WHvander. Species divergence and trait convergence in experimental plant community assembly. Ecol Lett. 2005;8:1283-90

48. Attractor - from Wolfram MathWorld. https://mathworld.wolfram.com/Attractor. html. Accessed Jun 2021.

49. Scheffer M, Carpenter S, Foley JA, Folke C, Walker B. Catastrophic shifts in ecosystems. Nature. 2001;413:591-6.

50. Yuan H, Mei R, Liao J, Liu W-T. Nexus of Stochastic and Deterministic Processes on Microbial Community Assembly in Biological Systems. Front Microbiol. 2019;10:1536.

51. Evans S, Martiny JBH, Allison SD. Effects of dispersal and selection on stochastic assembly in microbial communities. ISME J. 2017;11:176-85.

52. Leibold MA, Mcpeek MA. Coexistence Of The Niche And Neutral Perspectives In Community Ecology. Ecology. 2006;87:1399-410.

53. Sloan WT, et al. Quantifying the roles of immigration and chance in shaping prokaryote community structure. Environ Microbiol. 2006;8:732-40.

54. Fuhrman JA, Hagström Å. Bacterial and Archaeal Community Structure and its Patterns. Microbial Ecology of the Oceans: Second Edition. 2008; 45-90.

55. Andrews J, Harris R. r- and K-Selection and Microbial Ecology. Adv Microbial Ecol. 1986;9:99-147.

56. Vadstein O, Attramadal KJK, Bakke I, Olsen Y. K-Selection as Microbial Community Management Strategy: A Method for Improved Viability of Larvae in Aquaculture. Front Microbiol. 2018;9:2730.

57. Liu S, Wawrik B, Liu Z. Different Bacterial Communities Involved in Peptide Decomposition between Normoxic and Hypoxic Coastal Waters. Front Microbiol. 2017;0:353.

58. Baker-Austin C, et al. Vibrio spp. infections. Nat Rev Disease Primers. 2018:4:1-19.

59. Coutinho $\mathrm{FH}$, et al. Niche distribution and influence of environmental parameters in marine microbial communities: a systematic review. PeerJ. 2015;3:e1008.

60. Tromas N, et al. Niche Separation Increases With Genetic Distance Among BloomForming Cyanobacteria. Front Microbiol. 2018;0:438.

61. Miao $L$, et al. Distinct community structure and microbial functions of biofilms colonizing microplastics. Sci Total Environ. 2019;650:2395-402.

\section{ACKNOWLEDGEMENTS}

This study was part of the ERA-Net COFASP project "MicStaTech", funded by the Research Council of Norway (Contract 247558). Financial support was also provided by NTNU, Faculty of Natural Sciences, as a PhD scholarship to MSG and a Fulbright scholarship to IAM. We would like to thank T. Frede Thingstad and the Brendan Bohannan group for valuable comments on an earlier draft of this manuscript.

\section{AUTHOR CONTRIBUTIONS}

$I M$, IB, and OV designed the study. MG and IM conducted the study. MG analysed the data with input from TA and OV. MG prepared the initial draft of the manuscript before all authors contributed to writing and editing the final manuscript.

\section{COMPETING INTERESTS}

The authors declare no competing interests.

\section{ADDITIONAL INFORMATION}

Supplementary information The online version contains supplementary material available at https://doi.org/10.1038/s43705-021-00058-4.

Correspondence and requests for materials should be addressed to Madeleine $\mathrm{S}$. Gundersen.

Reprints and permission information is available at http://www.nature.com/ reprints

Publisher's note Springer Nature remains neutral with regard to jurisdictional claims in published maps and institutional affiliations.

\begin{abstract}
(i) Open Access This article is licensed under a Creative Common Attribution 4.0 International License, which permits use, sharing, adaptation, distribution and reproduction in any medium or format, as long as you give appropriate credit to the original author(s) and the source, provide a link to the Creative Commons licence, and indicate if changes were made. The images or other third party material in this article are included in the article's Creative Commons licence, unless indicated otherwise in a credit line to the material. If material is not included in the article's Creative Commons licence and your intended use is not permitted by statutory regulation or exceeds the permitted use, you will need to obtain permission directly from the copyright holder. To view a copy of this licence, visit http://creativecommons. org/licenses/by/4.0/.
\end{abstract}

(c) The Author(s) 2021, corrected publication 2022 